\title{
Experimental Evaluation of a Game-Theoretic Human Driver Steering Control Model
}

\author{
Xiaoxiang Na and David J. Cole
}

\begin{abstract}
Automated vehicle steering control systems have great potential to improve road safety. Development of such systems calls for mathematical driver models able to represent human drivers' steering behavior in response to automated steering intervention. This paper concerns experimental evaluation of a game-theoretic driver steering control model. The driver model centers on a steering control strategy developed based on the Nash equilibrium of a theoretic noncooperative game between driver and automated steering controller. Key parameters of the gametheoretic driver model are identified by fitting the model to real driver steering behavior measured from six driver subjects in an experiment using a driving simulator. The game-theoretic driver model is evaluated by comparing to a 'conventional' optimalcontrol-theoretic driver model, and analyzing their model fitting errors. Results from the analysis demonstrate that the gametheoretic driver model is statistically significantly better than the 'conventional' driver model for representing three out of the six subjects' steering behavior. For the other three subjects, both the two models perform statistically equivalently well.
\end{abstract}

Index Terms - Game theory, driver model, automated steering, experiment, evaluation

\section{INTRODUCTION}

$\mathrm{T}$ HE increasing demand for road safety is driving the need for advanced driver assistance systems. Sophisticated systems able to apply automatic braking have already been launched in cars. Such systems, however, may be less effective in avoiding collision with obstacles appearing suddenly in front of a vehicle, e.g. a pedestrian dashing onto road. In such situations, evasive steering maneuvers are necessary [1]. To this end, automated steering control is applicable [2], which generally involves four steps: first, obstacles are detected using onboard sensors; second, the risk of collision is assessed; third, a collision-free path is planned when the threat of an impending collision is identified; and finally, steering action is applied automatically to control the vehicle to follow the planned path. The actuation apparatus applying automated steering can be an Active Front Steering (AFS) system. This system is capable of superposing upon the steering column of a vehicle an additional angle that is independent of the driver's steering wheel angle. Applications of AFS systems to collision avoidance have been reported in several studies, for example, by Anderson et al. [3], Isermann et al. [4] and Turri et al. [5]. Despite its potential for improving road safety, AFS-based automated steering may give rise to undesirable driver reaction: as the collision-free path is planned

Manuscript received Xxxxxx, XX, 20XX; revised Xxxxxx, XX, 20XX; accepted Xxxxxx, XX, 20XX. The Associate Editor for this article was X. Xxxx. (Corresponding author: Xiaoxiang Na.)

The authors are with the Department of Engineering, University of Cambridge, CB2 1PZ, Cambridge, U.K. (e-mail: xnhn2@cam.ac.uk; djc13@cam.ac.uk). without knowledge of a driver's real intention, there is the possibility that the planned path differs from or even conflicts with the driver's target path. As a result, the two controllers, i.e. the AFS controller and the driver may compete for control. This may jeopardize the vehicle's obstacle avoidance performance.

Investigation of drivers' interaction with vehicle automation currently relies heavily on experimental methods involving test drivers $[6,7]$. As a result, technology development is expensive and time-consuming. In the automotive engineering process, validated mathematical models of vehicles were used to predict the effect of design changes from 1950s. There has been a need for mathematical models of human drivers that can operate in closed loop with vehicle models to evaluate the performance of the driver-vehicle system - so as to support decision-making in low-cost design phases. Such a driver model can be regarded as a 'virtual test driver' [8]. A thorough review of driver models applied to vehicle dynamics testing was given in [9]. Markkula [10] estimated that using a driver model to evaluate a vehicle active safety system in a computer simulation environment was approximately 100 times more cost-effective (in terms of cost incurred per test driving scenario) than using human drivers to conduct test-track tests. However, this requires a driver model that has been extensively validated in the driving scenarios of interest. Over the past decades, considerable efforts have been made to research and validate driver steering control models, where models developed using optimal control theory were found capable of representing human driver steering behavior in various driving scenarios, including path-following [11-13], resistance to crosswind [14], reaction to steering system failure [15] etc. In these applications, there was only one controller, i.e. the human driver. It is therefore a question whether the optimalcontrol-theoretic driver models are suitable to represent driver steering behavior in scenarios where two controllers, i.e. driver and vehicle automated steering system act at the same time.

In the research arena of human-agent interaction, game theory was found an effective approach to modeling human's interplay with intelligent agents in several circumstances. Of particular note here is the work by Braun et al. [16] who studied human players' arm movements in a virtual two-person ropepulling game. A game-theoretic model was built up, and its Nash solution was used to predict human players' arm movements. A high degree of agreement was seen between model output and measured player arm movements. Quite recently, Schmid and Braun [17] revisited their work on human sensorimotor interactions [16, 18-19] and remarked that human players in simple sensorimotor games acted in accordance with simulation results derived from noncooperative game theory. In separate studies, Chackochan and Sanguineti [20] developed a noncooperative-game-theoretic model to predict human players' arm forces in an arm reaching game, and validated it against experiments. Li et al. [21] reported a noncooperative game- 


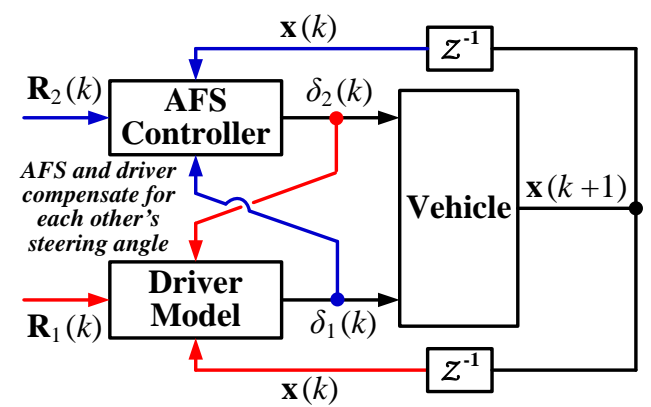

Fig. 1. Driver-AFS noncooperative steering control scheme

theoretic algorithm able to identify human operators' control behavior when interacting with robots in arm reaching tasks. Findings from these studies support the remark made by Braun et al. that human sensorimotor interaction displayed in simple games can be represented using game-theoretic models.

Narrowing down into the arena of driver-vehicle interaction, there are a few pioneering studies discussing the application of game theory to modeling driver control behavior. Flad et al. [22] proposed a noncooperative-game model to predict driver steering torque in response to the steering control applied by an advanced driver assistance system. $\mathrm{Na}$ and Cole [23] proposed a game-theoretic paradigm to predict driver steering angle in response to vehicle automated steering control. Tamaddoni et al. [24] reported a vehicle stability controller based on the assumption that the controller's stabilizing moment and the driver's steering action reach a Nash equilibrium. Dextreit and Kolmanovsky [25] developed a vehicle energy management algorithm where the control command is determined by solving a Stackelberg game between the driver the controller. Ji et al. [26] proposed a driver-automation shared control scheme, where a noncooperative-game-theoretic model was applied to estimate the driver's steering control behavior in response to the intervention from vehicle automation. Quite recently, Tian et al. [27] proposed a game-theoretic approach to modeling the interactions among human-driven and autonomous vehicles in urban traffic, where the decision-making processes of drivers and vehicles were formulated based on level-k game theory.

The works reviewed above imply that game theory could be suitable for modeling human drivers' interaction with vehicle automation. However, it is a question whether a game-theoretic model is necessary, in comparison to a 'conventional' optimalcontrol-theoretic model. If the answer is yes, another question follows: to what extent a game-theoretic model is advantageous over a 'conventional' model? This paper aims to evaluate the advantage of the game-theoretic driver steering control model proposed by the authors in [23] in representing driver steering behavior in a path-following scenario that involves driver and vehicle automation compete for steering control. The steering behavior of six subjects is measured on a fixed-base driving simulator and used to identify the game-theoretic driver model. The work is thought to be among the first efforts to evaluate game-theoretic driver models using measured driver control behavior. Such models may allow deeper insights into the cognitive behavior of human drivers, so that optimization of present or future driver-automation interfaces, e.g. force control steering [28] and steering-by-wire [29] becomes a possibility.
TABLE I

VEHICLE MODEL PARAMETERS

\begin{tabular}{lll}
\hline \hline Symbol & Description & Value and Unit \\
\hline$m$ & vehicle mass & $1840 \mathrm{~kg}$ \\
$I$ & vehicle yaw moment of inertia & $3000 \mathrm{kgm}^{2}$ \\
$a / b$ & $\begin{array}{l}\text { distance from vehicle centre of } \\
\text { mass to front axle / rear axle }\end{array}$ & $1.136 / 1.663 \mathrm{~m}$ \\
& Tire cornering stiffness of & $116000 / 187000 \mathrm{~N} / \mathrm{rad}$ \\
$C_{\mathrm{f}} / C_{\mathrm{r}}$ & front / rear axle & \\
& vehicle steering ratio & 15.8 \\
\hline \hline
\end{tabular}

The remainder of the paper is structure as follows. Section II describes the derivation of driver and AFS steering strategies in the scheme of driver-AFS noncooperative control. Section III reports the driving simulator experiment for measuring human driver steering behavior. Section IV presents the method and procedure for fitting driver steering control models to measured driver steering behavior. Section V describes the model fitting results and discusses the advantage of the game-theoretic driver model in representing human driver steering behavior. Section $\mathrm{V}$ draws conclusions and advises future work.

\section{DRIVER-AFS NonCOOPERATIVE STEERING CONTROL SCHEME}

Fig. 1 shows the driver-AFS noncooperative steering control scheme, which was proposed by the authors in [23]. It outlines how a driver is modelled to interact with an AFS controller in the context of a noncooperative game, where the driver and the AFS controller hold individual target paths $\mathbf{R}_{1}(k)$ and $\mathbf{R}_{2}(k)$, respectively. The AFS controller determines its steering angle $\delta_{2}(k)$ at time step $k$ using its target path $\mathbf{R}_{2}(k)$, vehicle state $\mathbf{x}(k)$, and the driver's steering angle $\delta_{1}(k)$. Note that the AFS controller is designed to partially compensate for the driver's steering angle $\delta_{1}(k)$, so as to mitigate adverse impacts due to driver error. Such a design was implemented in several works, e.g. [3] and [30]. The driver is modelled to determine his or her steering angle $\delta_{1}(k)$ by taking $\mathbf{x}(k), \mathbf{R}_{1}(k)$, and AFS steering angle $\delta_{2}(k)$ into account. In this section, the driver's and the AFS controller's steering strategies will be derived.

\section{A. Vehicle Dynamics}

The 'Vehicle' module shown in Fig. 1 receives the driver's and the AFS controller's steering angles $\delta_{1}(k)$ and $\delta_{2}(k)$, and effects a change in vehicle state from $\mathbf{x}(k)$ to $\mathbf{x}(k+1)$. To reduce the complexity involved in the derivation of the gametheoretic driver model, the vehicle is assumed to operate in linear regime at a constant longitudinal velocity. Hence, the linear time-invariant 'bicycle' model described in [23] is used. The continuous-time representation of the model is: 


$$
\begin{aligned}
\left\{\begin{array}{c}
\dot{v}(t) \\
\dot{\omega}(t) \\
\dot{y}(t) \\
\dot{\psi}(t)
\end{array}\right\} & {\left[\begin{array}{cccc}
\frac{-\left(C_{\mathrm{f}}+C_{\mathrm{r}}\right)}{U m} & \frac{-\left(a C_{\mathrm{f}}-b C_{\mathrm{r}}\right)}{U m}-U & 0 & 0 \\
\frac{-\left(a C_{\mathrm{f}}-b C_{\mathrm{r}}\right)}{U I} & \frac{-\left(a^{2} C_{\mathrm{f}}+b^{2} C_{\mathrm{r}}\right)}{U I} & 0 & 0 \\
1 & 0 & 0 & U \\
0 & 1 & 0 & 0
\end{array}\right]\left\{\begin{array}{c}
v(t) \\
\omega(t) \\
y(t) \\
\psi(t)
\end{array}\right\} } \\
& +\left[\begin{array}{c}
\frac{C_{\mathrm{f}}}{m G} \\
\frac{a C_{\mathrm{f}}}{I G} \\
0 \\
0
\end{array}\right] \delta_{1}(t)+\left[\begin{array}{c}
\frac{C_{\mathrm{f}}}{m G} \\
\frac{a C_{\mathrm{f}}}{I G} \\
0 \\
0
\end{array}\right] \delta_{2}(t)
\end{aligned}
$$

where $v(t)$ is the lateral velocity of the vehicle; $\omega(t)$ the yaw rate; $y(t)$ the lateral displacement; $\psi(t)$ the yaw angle; $U$ the longitudinal velocity; $m$ the mass; $I$ the polar inertia; $a$ the distance from center of mass to front axle; $b$ the distance from center of mass to rear axle; $C_{\mathrm{f}}$ the front axle cornering stiffness; $C_{\mathrm{r}}$ the rear axle cornering stiffness; and $G$ the steering gear ratio. Values of the vehicle model parameters used in this paper are provided in Table I. The continuous-time model (1) can be discretized using Matlab function 'c2d'. This results in:

$$
\mathbf{x}_{\mathrm{v}}(k+1)=\mathbf{A}_{\mathrm{v}} \mathbf{x}_{\mathrm{v}}(k)+\mathbf{B}_{\mathrm{v} 1} \delta_{1}(k)+\mathbf{B}_{\mathrm{v} 2} \delta_{2}(k)
$$

where $\mathbf{x}_{\mathrm{v}}(k)=\left\{\begin{array}{llll}v(k) & \omega(k) & y(k) & \psi(k)\end{array}\right\}^{\mathrm{T}}$ is the vehicle state vector at time step $k . \mathbf{x}_{\mathrm{v}}(k)$ is then extended to incorporate a new state $y_{\text {int }}(k)$ which denotes the time integral of the lateral displacement, i.e. $y_{\text {int }}(k+1)=y_{\text {int }}(k)+y(k)$. As a result, the discrete-time representation of vehicle dynamics becomes:

$$
\mathbf{x}(k+1)=\mathbf{A} \mathbf{x}(k)+\mathbf{B}_{1} \delta_{1}(k)+\mathbf{B}_{2} \delta_{2}(k)
$$

where $\mathbf{x}(k)=\left\{\begin{array}{lllll}v(k) & \omega(k) & y(k) & \psi(k) \quad y_{\text {int }}(k)\end{array}\right\}^{\mathrm{T}}$ is the state vector; $\mathbf{A}$ the state matrix; $\mathbf{B}_{1}$ and $\mathbf{B}_{2}$ the input matrices. The output vector is defined as $\mathbf{z}(k)=\left\{\begin{array}{llll}y(k) & y_{\text {int }}(k) & \psi(k)\end{array}\right\}^{\mathrm{T}}$. Here, $y(k)$ is included in $\mathbf{z}(k)$ to allow either the driver or the AFS controller to penalize lateral displacement error; $y_{\text {int }}(k)$ is included to penalize the integral of lateral displacement error; and $\psi(k)$ is to penalize the derivative of lateral displacement error. Note that $\psi(k)$ can be determined using (4) below, as suggested in [31]:

$$
\psi(k)=\frac{y(k+1)-y(k)}{U \cdot T}
$$

where $T$ denotes the sampling time in the discrete system (3).

\section{B. Driver and AFS Steering Strategies}

The steering strategies of the driver and the AFS controller are derived based on the formulation made in [23]. A solution technique for decoupling the two strategies is provided below.

First, the driver's prediction equation is constructed by iterating (3) for $N_{1}$ times ahead, which results in (5):

$$
\mathbf{Z}_{1}(k)=\boldsymbol{\Psi}_{1} \mathbf{x}(k)+\boldsymbol{\Theta}_{1} \delta_{1}(k)+\mathbf{\Omega}_{1} \delta_{2}(k)
$$

where

$$
\mathbf{Z}_{1}(k)=\left\{\begin{array}{lllll}
\mathbf{z}(k) & \mathbf{z}(k+1) & \mathbf{z}(k+2) & \cdots & \mathbf{z}\left(k+N_{1}\right)
\end{array}\right\}^{\mathrm{T}},
$$

$$
\begin{gathered}
\boldsymbol{\Psi}_{1}=\left[\begin{array}{lllll}
\mathbf{C} & \mathbf{C A} & \mathbf{C A}^{2} & \cdots & \mathbf{C A}^{N_{1}}
\end{array}\right]^{\mathrm{T}}, \\
\boldsymbol{\Theta}_{1}=\left[\begin{array}{lllll}
\mathbf{0} & \mathbf{C B}_{1} & \sum_{i=0}^{1} \mathbf{C A}^{i} \mathbf{B}_{1} & \cdots & \sum_{i=0}^{N_{1}-1} \mathbf{C A}^{i} \mathbf{B}_{1}
\end{array}\right]^{\mathrm{T}}, \text { and } \\
\boldsymbol{\Omega}_{1}=\left[\begin{array}{llllll}
\mathbf{0} & \mathbf{C B}_{2} & \sum_{i=0}^{1} \mathbf{C A}^{i} \mathbf{B}_{2} & \cdots & \sum_{i=0}^{N_{1}-1} \mathbf{C A}^{i} \mathbf{B}_{2}
\end{array}\right]^{\mathrm{T}} .
\end{gathered}
$$

Here $N_{1}$ represents the driver's preview horizon. During the iteration of (3) over $N_{1}$ times, the driver steering angle $\delta_{1}(k)$ is kept unchanged. In other words, the driver's control horizon is unity. Such a treatment is adopted according to the observation made in [11] that a human driver is less likely to change their steering action over preview horizon. $\mathbf{Z}_{1}(k)$ contains vehicle outputs from $\mathbf{z}(k)$ up to $\mathbf{z}\left(k+N_{1}\right)$, where $\mathbf{z}(k+j)$ for $j=0,1,2 \ldots, N_{1}$ represents the driver's predicted vehicle output at future time step $k+j$, that is

$$
\mathbf{z}(k+j)=\left\{\begin{array}{lll}
y(k+j) & y_{\text {int }}(k+j) \quad \psi(k+j)
\end{array}\right\}^{\mathrm{T}} .
$$

$\boldsymbol{\Psi}_{1}, \boldsymbol{\Theta}_{1}$ and $\boldsymbol{\Omega}_{1}$ are matrices of appropriate dimensions.

Second, the driver's cost function is defined as:

$$
J_{1}(k)=\left[\mathbf{Z}_{1}(k)-\mathbf{R}_{1}(k)\right]^{\mathrm{T}} \mathbf{Q}_{1}\left[\mathbf{Z}_{1}(k)-\mathbf{R}_{1}(k)\right]+\delta_{1}(k)^{2}
$$

In (6), the driver's path-following error is quantified as the difference between the driver's predicted vehicle output $\mathbf{Z}_{1}(k)$ and target path $\mathbf{R}_{1}(k)$, i.e. $\mathbf{Z}_{1}(k)-\mathbf{R}_{1}(k)$. Because the driver's predicted output $\mathbf{z}(k+j)$ appearing in $\mathbf{Z}_{1}(k)$ is composed of $y(k+j), y_{\text {int }}(k+j)$ and $\psi(k+j)$, the target path $\mathbf{R}_{1}(k)$ can be defined in an analogous format to $\mathbf{z}(k+j)$ such that:

$$
\mathbf{r}_{1}(k+j)=\left\{\begin{array}{lll}
r_{1}^{y}(k+j) & r_{1}^{y_{\text {int }}}(k+j) & r_{1}^{\psi}(k+j)
\end{array}\right\}^{\mathrm{T}} .
$$

where $r_{1}^{y}(k+j)$ is the target lateral displacement of the driver, $r_{1}^{y_{\text {int }}}(k+j)$ the target lateral integral, and $r_{1}^{\psi}(k+j)$ the target yaw angle.

$\mathbf{Q}_{1}$ in (6) is a diagonal matrix of $\mathbf{q}_{1}$, and $\mathbf{q}_{1}$ is composed of the driver's path-error weights $q_{1}^{y}, q_{1}^{y_{\text {itt }}}$ and $q_{1}^{\psi}$ :

$$
\mathbf{Q}_{1}=\left[\begin{array}{ccc}
\mathbf{q}_{1} & \cdots & \mathbf{0} \\
\vdots & \ddots & \vdots \\
\mathbf{0} & \cdots & \mathbf{q}_{1}
\end{array}\right], \mathbf{q}_{1}=\left[\begin{array}{ccc}
q_{1}^{y} & 0 & 0 \\
0 & q_{1}^{y_{\text {int }}} & 0 \\
0 & 0 & q_{1}^{\psi}
\end{array}\right] .
$$

Here $q_{1}^{y}, q_{1}^{y_{\text {int }}}$ and $q_{1}^{\psi}$ are respectively used to weigh the driver's lateral displacement error $y(k+j)-r_{1}^{y}(k+j)$, lateral displacement integral error $y_{\text {int }}(k+j)-r_{1}^{y_{\text {int }}}(k+j)$ and yaw angle error $\psi(k+j)-r_{1}^{\psi}(k+j)$.

The driver steering angle $\delta_{1}(k)$ that minimizes cost function (6) can be then obtained through solving a least-squares problem. This involves the following procedure:

Substituting prediction equation (5) into (6) yields:

$$
J_{1}(k)=\left[\boldsymbol{\Theta}_{1} \delta_{1}(k)-\boldsymbol{\varepsilon}_{1}(k)\right]^{\mathrm{T}} \mathbf{Q}_{1}\left[\boldsymbol{\Theta}_{1} \delta_{1}(k)-\boldsymbol{\varepsilon}_{1}(k)\right]+\delta_{1}(k)^{2}
$$

where $\boldsymbol{\varepsilon}_{1}(k)$ is an intermediate term which is define as:

$$
\boldsymbol{\varepsilon}_{1}(k)=\mathbf{R}_{1}(k)-\left[\boldsymbol{\Psi}_{1} \mathbf{x}(k)+\mathbf{\Omega}_{1} \delta_{2}(k)\right]
$$

(7) can be then rewritten as:

$$
J_{1}(k)=\left\{\begin{array}{c}
\mathbf{S}_{1}\left[\boldsymbol{\Theta}_{1} \delta_{1}(k)-\boldsymbol{\varepsilon}_{1}(k)\right] \\
\delta_{1}(k)
\end{array}\right\}\left\{\begin{array}{c}
\mathbf{S}_{1}\left[\boldsymbol{\Theta}_{1} \delta_{1}(k)-\boldsymbol{\varepsilon}_{1}(k)\right] \\
\delta_{1}(k)
\end{array}\right\}
$$

where $\mathbf{S}_{1}$ is a diagonal matrix, each element of which is the 


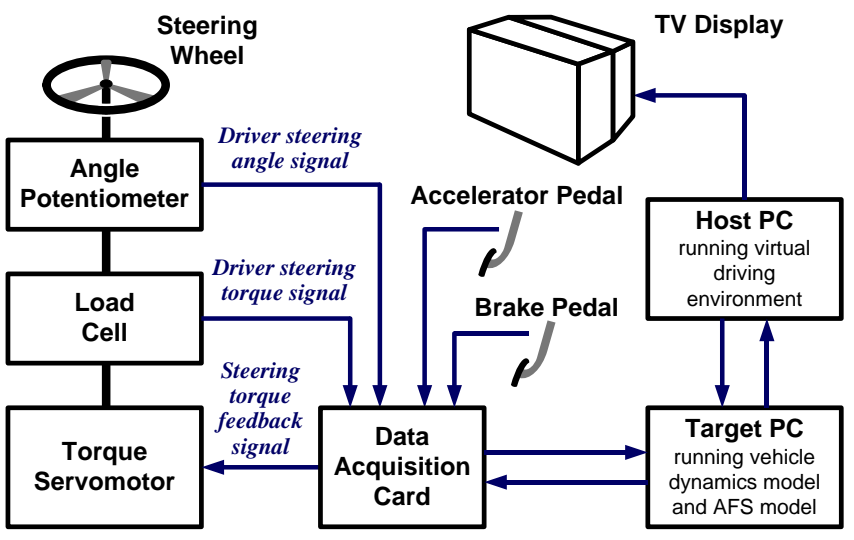

(a)

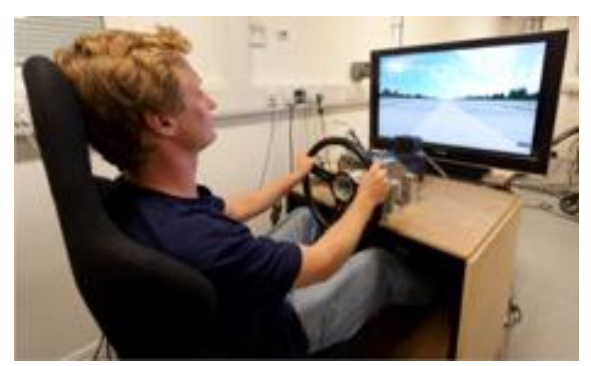

(b)

Fig. 2. A fixed-base driving simulator used for experiment: (a) Configuration scheme. (b) Driver interface.

square root of corresponding one in $\mathbf{Q}_{1}$, i.e. $\mathbf{S}_{1}=\operatorname{sqrt}\left(\mathbf{Q}_{1}\right)$. Hence, the driver steering angle $\delta_{1}(k)$ that minimizes (6), or equivalently, (7) is the least-square solution of:

$$
\left[\begin{array}{c}
\mathbf{S}_{1} \boldsymbol{\Theta}_{1} \\
1
\end{array}\right] \delta_{1}(k)=\left[\begin{array}{c}
\mathbf{S}_{1} \\
0
\end{array}\right] \boldsymbol{\varepsilon}_{1}(k)
$$

In view of the structure of (10), the driver steering angle $\delta_{1}(k)$ can be solved numerically using $\mathrm{QR}$ decomposition. This gives:

$$
\delta_{1}(k)=\mathbf{K}_{1} \boldsymbol{\varepsilon}_{1}(k)
$$

where $\mathbf{K}_{1}$ is the matrix resulted from the $\mathrm{QR}$ decomposition.

Now, substituting (8) back to (11) yields:

$$
\delta_{1}(k)=\mathbf{G}_{1}\left\{\begin{array}{c}
\mathbf{x}(k) \\
\mathbf{R}_{1}(k)
\end{array}\right\}+\mathbf{L}_{1} \delta_{2}(k)
$$

Gain arrays $\mathbf{G}_{1}$ and $\mathbf{L}_{1}$ in (12) respectively take the form of $\mathbf{G}_{1}=\left[\begin{array}{ll}-\mathbf{K}_{1} \boldsymbol{\Psi}_{1} & \mathbf{K}_{1}\end{array}\right]$ and $\mathbf{L}_{1}=-\mathbf{K}_{1} \boldsymbol{\Omega}_{1}$. Both are functions of $\mathbf{A}$, $\mathbf{B}_{1}, \mathbf{B}_{2}, \mathbf{C}, N_{1}, q_{1}^{y}, q_{1}^{y_{\text {int }}}$ and $q_{1}^{\psi}$.

The AFS controller's steering strategy can be derived following a similar procedure. This starts from constructing the AFS controller's prediction equation (13), as below:

$$
\mathbf{Z}_{2}(k)=\boldsymbol{\Psi}_{2} \mathbf{x}(k)+\boldsymbol{\Theta}_{2} \delta_{1}(k)+\boldsymbol{\Omega}_{2} \delta_{2}(k)
$$

Note that (13) is derived by iterating (3) for $N_{2}$ times ahead, where $N_{2}$ is the AFS controller's preview horizon. Hence $\boldsymbol{\Psi}_{2}$, $\boldsymbol{\Theta}_{2}$ and $\boldsymbol{\Omega}_{2}$ in (13) hold similar structures to $\boldsymbol{\Psi}_{1}, \boldsymbol{\Theta}_{1}$ and $\boldsymbol{\Omega}_{1}$ in (5), respectively. $N_{2}$ reflects how far the AFS system is able to see ahead. It is subject to the detection range of the on-board
TABLE II

EXPERIMENT SUBJECT DETAILS

\begin{tabular}{cccc}
\hline \hline Subject No. & Gender & Age & Driving Experience (years) \\
\hline 1 & Female & 26 & 3 \\
2 & Male & 34 & 12 \\
3 & Male & 27 & 4 \\
4 & Male & 25 & 5 \\
5 & Female & 26 & 1 \\
6 & Male & 24 & 4 \\
\hline \hline
\end{tabular}

sensor in use, e.g. a camera or lidar. $N_{2}$ is independent of driver preview horizon $N_{1}$, and can be set larger than $N_{1}$. This enables the AFS target path $\mathbf{R}_{2}(k)$ to extend further than driver target path $\mathbf{R}_{1}(k)$. As a result, the AFS controller can apply control actions in more advance. During deriving (13), the AFS angle $\delta_{2}(k)$ is kept unchanged. This setting was used in several control systems to reduce computational burden, e.g. in [32].

Similar to the driver's cost function (6), the AFS controller's cost function can be constructed as:

$$
J_{2}(k)=\left[\mathbf{Z}_{2}(k)-\mathbf{R}_{2}(k)\right]^{\mathrm{T}} \mathbf{Q}_{2}\left[\mathbf{Z}_{2}(k)-\mathbf{R}_{2}(k)\right]+\delta_{2}(k)^{2}
$$

Similar to $\mathbf{Q}_{1}$ in (6), $\mathbf{Q}_{2}$ is a diagonal matrix parameterized by the AFS controller's path-error weights $q_{2}^{y}, q_{2}^{y_{\text {int }}}$ and $q_{2}^{\psi}$.

Minimizing AFS cost function (14) subject to its prediction equation (13) gives the AFS controller's steering strategy:

$$
\delta_{2}(k)=\mathbf{G}_{2}\left\{\begin{array}{c}
\mathbf{x}(k) \\
\mathbf{R}_{2}(k)
\end{array}\right\}+\mathbf{L}_{2} \delta_{1}(k)
$$

where $\mathbf{G}_{2}$ and $\mathbf{L}_{2}$ are gain arrays having similar structures to $\mathbf{G}_{1}$ and $\mathbf{L}_{1}$ shown in (12), respectively. $\mathbf{G}_{2}$ and $\mathbf{L}_{2}$ are both functions of $\mathbf{A}, \mathbf{B}_{1}, \mathbf{B}_{2}, \mathbf{C}, N_{2}, q_{2}^{y}, q_{2}^{y_{\text {int }}}$ and $q_{2}^{\psi}$.

It can be seen that the driver steering angle $\delta_{1}(k)$ determined by (12) linearly depends on the AFS angle $\delta_{2}(k)$, while the AFS angle $\delta_{2}(k)$ determined by (15) linearly depends on the driver angle $\delta_{1}(k)$. In other words, (12) and (15) are coupled. For this issue, two remarks are made as below.

First, the driver angle $\delta_{1}(k)$ given by (12) minimizes driver cost function (6) in response to a specific AFS angle $\delta_{2}(k)$. It is not clear at the moment whether (12) has a Nash equilibrium feature. To investigate this point, (15) is substituted into (12) which yields

$$
\delta_{1}(k)=\left[\mathbf{I}-\mathbf{L}_{1} \mathbf{L}_{2}\right]^{-1} \mathbf{G}_{1}\left\{\begin{array}{c}
\mathbf{x}(k) \\
\mathbf{R}_{1}(k)
\end{array}\right\}+\left[\mathbf{I}-\mathbf{L}_{1} \mathbf{L}_{2}\right]^{-1} \mathbf{L}_{1} \mathbf{G}_{2}\left\{\begin{array}{c}
\mathbf{x}(k) \\
\mathbf{R}_{2}(k)
\end{array}\right\}
$$

In (16), AFS angle $\delta_{2}(k)$ vanishes. This implies that the driver angle $\delta_{1}(k)$ determined by (16) minimizes driver cost function (6) with respect to any AFS angle. Such a feature, observed by Rawlings and Mayne [33], is a Nash equilibrium. Hence, (16) explicitly denotes the driver's Nash steering strategy. The driver angle $\delta_{1}(k)$ given by (16) is numerically the same as that determined by (12), given that the AFS controller sticks to its strategy (15). This implies that (12) is numerically equivalent to (16), though it does not explicitly show a Nash equilibrium. 


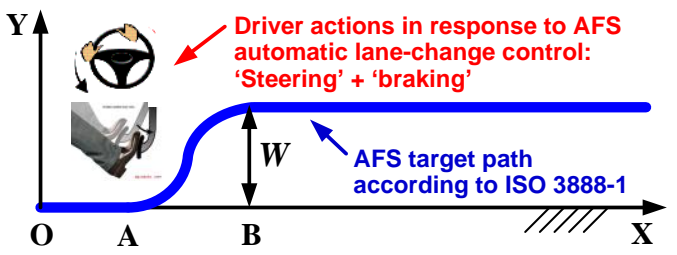

Fig. 3. Profile of AFS lane-change target path used in trials.

Hence, (12) and (16) are equivalently the driver's Nash steering strategy. As (12) takes a more concise format, it will be used in the simulation and model fitting in this paper.

Second, the coupling between the driver's Nash steering strategy (12) and the AFS controller's strategy (15) can be solved by amending (15) to (17):

$$
\delta_{2}(k)=\mathbf{G}_{2}\left\{\begin{array}{c}
\mathbf{x}(k) \\
\mathbf{R}_{2}(k)
\end{array}\right\}+\mathbf{L}_{2} \delta_{1}(k-1)
$$

In (17), the AFS angle $\delta_{2}(k)$ now depends on the driver angle determined at time step $k-1$. Such an amendment causes little change to $\delta_{2}(k)$, given that the sampling time $T$ of the discrete system is sufficiently small. In this paper, $T=0.01 \mathrm{~s}$ is used. It was found in [34] that this sampling time resulted in negligible difference between the AFS angle calculated by (17) and (15).

Based on (17), the simulation of driver steering control can be implemented following such a procedure: at time step $k$, the AFS controller uses (17) to determine its steering angle $\delta_{2}(k)$; the driver model then uses (12) to determine its Nash steering angle $\delta_{1}(k)$; vehicle state $\mathbf{x}(k)$ is then updated using (3). At the next time step, the procedure repeats. At this point, the question whether the driver's Nash steering strategy (12) can represent human drivers' steering behavior in response to the intervention of the AFS controller (15) rises. To answer this, the driver Nash strategy (12) will be fitted to measured driver steering behavior in response to AFS controller (15). In the next section, an experiment using a driving simulator for measuring human driver steering behavior will be described.

\section{DRIVING SimULATOR EXPERIMENT}

This section describes the design of the driving simulator experiment for measuring human driver steering behavior.

\section{A. Experiment Apparatus}

A fixed-base driving simulator was used in the experiment. It was developed using Matlab Real-Time Workshop is. Fig. 2 (a) shows its configuration scheme: it consists of (i) a target PC running vehicle model (3) and AFS controller (17); (ii) a host PC running virtual driving scenario; (iii) a TV displaying the virtual driving scenario to a test driver; (iv) a steering wheel allowing the driver to apply steering control to vehicle model (3); (v) an potentiometer measuring steering wheel angle; (vi) a load cell measuring driver steering torque; (vii) a servomotor offering steering torque feedback to driver; (viii) an accelerator and a brake pedal allowing the driver to change vehicle speed; (ix) a data acquisition card that logs and transmits data. Fig. 2 (b) shows the driver interface of the driving simulator.

\section{B. Experiment Subjects}

Six subjects marked as Subjects 1 to 6 were employed. Their details including gender, age and driving experience are given in Table II. It is recognized that the small number of subjects do not necessarily represent the general human driver population. However, the objective of this work is to investigate whether the game-theoretic driver model (12) can represent the control behavior of the six individual test subjects, and if (12) is better than an optimal-control-theoretic model (21) (to be described in Section IV) in response to the intervention of the AFS control by (17). At this stage, the objective is not to measure and model the control behavior of the general population of drivers. Such a work may follow if the gam-theoretic model is found a suitable candidate model for a larger measurement study.

\section{Experiment Preparation: AFS Controller Setup}

As advised previously, AFS controller (17) was programmed into the driving simulator. As shown in (17), the path-following performance of the AFS controller is dominated by its preview horizon $N_{2}$ and path-error weights $q_{2}^{y}, q_{2}^{y_{\text {int }}}$ and $q_{2}^{\psi}$. The determination of these four parameters is reported below.

The AFS controller's review horizon $N_{2}$ was set 200 steps (2.0 s), implying that the AFS system is able to see $40 \mathrm{~m}$ ahead, at a longitudinal velocity of $U=20 \mathrm{~m} / \mathrm{s}$. This conforms to the preview range of typical onboard cameras [4].

The AFS controller's yaw angle error weight $q_{2}^{\psi}$ was fixed at 0 , meaning that the vehicle yaw angle error is not penalized by the AFS controller. Such a setting was adopted because the control of vehicle lateral position is usually more significant than that of vehicle heading in collision avoidance scenarios.

The AFS controller's lateral displacement error weight $q_{2}^{y}$ and its lateral displacement error integral weight $q_{2}^{y_{\text {int }}}$ were determined via several trials prior to the experiment. Note that these trials were run for determining $q_{2}^{y}$ and $q_{2}^{y_{\text {itr }}}$ of the AFS controller exclusively. The experiment was started until proper $q_{2}^{y}$ and $q_{2}^{y_{\text {int }}}$ were found. In this paper, the term 'trial' denotes the activity for determining $q_{2}^{y}$ and $q_{2}^{y_{\text {int }}}$ while the term 'experiment' denotes the activity for measuring the six driver subjects' steering behavior in response to AFS control. Only the data measured in the experiment were used for model fitting and evaluation, which will be described in Section IV.

In a trial, the AFS controller (17) was loaded with a specific $q_{2}^{y}$. The properness of this $q_{2}^{y}$ was then assessed in a specific driving scenario where the AFS controller was programmed to conduct lane-change maneuvers automatically. The target path of the AFS controller is shown in Fig. 3. The profile is defined based on ISO 3888-1 [35], with $\mathrm{OA}=15 \mathrm{~m}, \mathrm{AB}=15 \mathrm{~m}$, and lane width $W=3.5 \mathrm{~m}$. Each of the six subjects detailed in Table II was then asked to counter the AFS lane-change control on the driving simulator by taking two actions simultaneously: (i) steering via the steering wheel to keep the vehicle travelling on its original lane; and (ii) braking via the brake pedal to stop the vehicle. Such a combined steering and braking is thought a common reaction of drivers to unexpected vehicle drifting.

For a specific $q_{2}^{y}$, each subject performs 12 trials, consisting of 6 leftward AFS lane-change maneuvers and 6 rightward ones. It can be imagined that larger $q_{2}^{y}$ results in more severe 

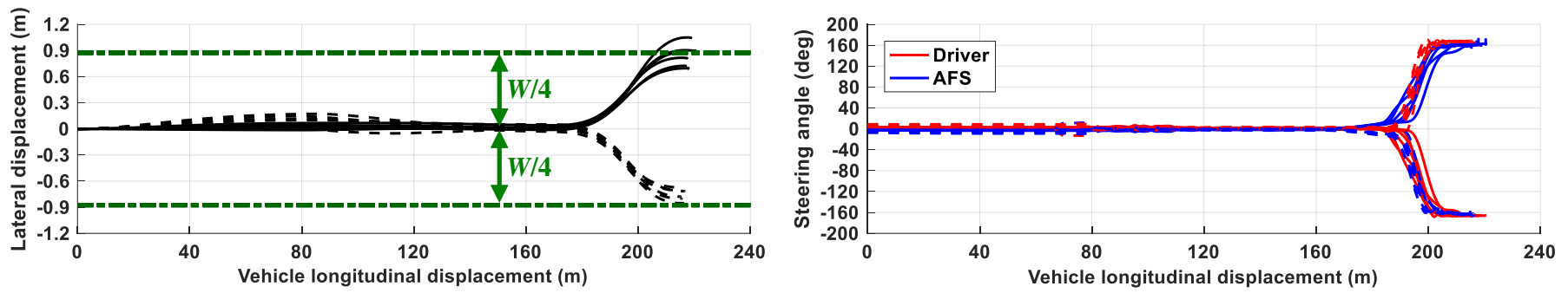

Fig. 4. Trial results of driver Subject 2, under $q_{2}^{y}=1.2 \mathrm{e}-3$. Solid lines: results from AFS controller performing leftwards lane change maneuvers; dashed lines: results from AFS controller performing rightward lane-change maneuvers.
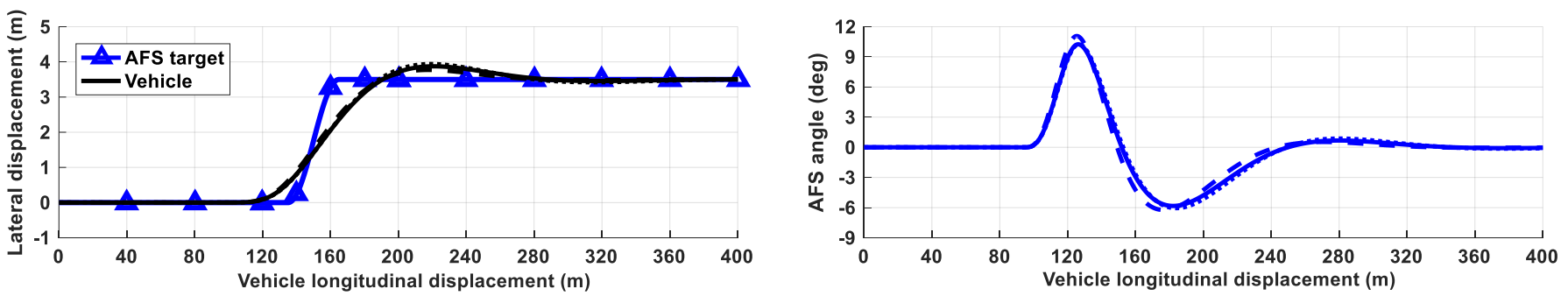

Fig. 5. Performance of AFS path-following control, when driver is not applying steering. Solid lines: AFS Weight Set 1: $q_{2}^{y}=0.6 \mathrm{e}-3, q_{2}^{y_{\text {int }}}=0$, and $q_{2}^{\psi}=0$; dashed lines: AFS Weight Set $2: q_{2}^{y}=1.2 \mathrm{e}-3, q_{2}^{y_{\mathrm{int}}}=0$, and $q_{2}^{\psi}=0$; dotted lines: AFS Weight Set 3: $q_{2}^{y}=0.6 \mathrm{e}-3, q_{2}^{y_{\text {ivt }}}=1.5 \mathrm{e}-8$, and $q_{2}^{\psi}=0$.
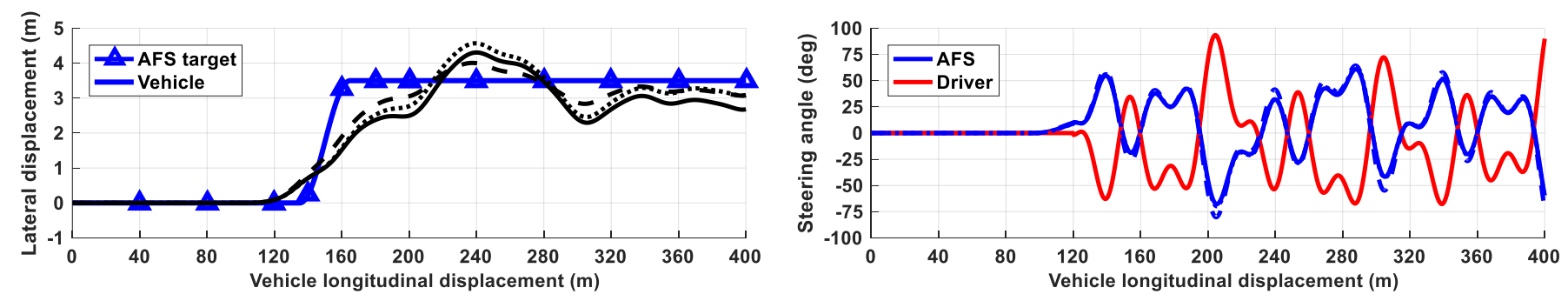

Fig. 6. Performance of AFS path-following control, when driver is applying disturbance steering. Solid lines: AFS Weight Set 1: $q_{2}^{y}=0.6 \mathrm{e}-3, q_{2}^{y_{\text {int }}}=0$, and $q_{2}^{\psi}=0$; dashed lines: AFS Weight Set $2: q_{2}^{y}=1.2 \mathrm{e}-3, q_{2}^{y_{\text {int }}}=0$, and $q_{2}^{\psi}=0$; dotted lines: AFS Weight Set 3: $q_{2}^{y}=0.6 \mathrm{e}-3, q_{2}^{y_{\text {ith }}}=1.5 \mathrm{e}-8$, and $q_{2}^{\psi}=0$.

AFS lane change. In response, the driver has to exert larger steering angles to counter the AFS control. This implies that there exist two conflicting requirements for $q_{2}^{y}$ :

1) Requirement on AFS path-following performance

$q_{2}^{y}$ should be adequately large to allow the AFS controller to deliver desirable path-following performance.

2) Requirement on driver overridability

$q_{2}^{y}$ should not be too large so that a driver still has the chance to override the AFS system, if the driver decides to do so.

When $q_{2}^{y}$ is set to be 0 , the AFS controller puts in no effort to control the vehicle. As a result, the second requirement can be met but the first requirement cannot. As $q_{2}^{y}$ increases, the AFS controller tends to give better path-following performance but the driver will find it increasingly difficult to override the AFS control. A $q_{2}^{y}$ compromising the two requirements can be determined by looking at the lateral displacement of the vehicle resulted from the steering interaction between driver and AFS in a trial. Specifically, the maximum $q_{2}^{y}$ that allows the driver to maintain the vehicle within a deviation of $W / 4$ from the lane center OX (shown in Fig. 3) is expected to be a proper value. Following a large number of trials, $q_{2}^{y}=1.2 \mathrm{e}-3$ was determined. Fig. 4 shows the trial results of Subject 2 under $q_{2}^{y}=1.2 \mathrm{e}-3$. The right plot shows the steering angles of the driver and the AFS. It can be seen that the AFS started lane change when the vehicle reached a longitudinal position of $170 \mathrm{~m}$. In response, the driver started to counter the AFS by steering in the opposite direction and applying brakes. The left plot shows the vehicle's lateral displacement resulted from the driver-AFS interaction. Specifically, those resulted from leftward AFS lane changes are marked in solid lines while those from rightward ones in dashed lines. The $\pm W / 4$ boundaries are marked in dash-dot lines. Note that data in Fig. 4 are presented until vehicle reached a full stop. It was found that this $1.2 \mathrm{e}-3$ is the largest $q_{2}^{y}$ that can allow all the six subjects listed in Table II to maintain the vehicle to stay within the $\pm W / 4$ boundaries in most cases.

Based on the results from the trials, an AFS controller weight set involving $q_{2}^{y}=1.2 \mathrm{e}-3, q_{2}^{y_{\text {int }}}=0$, and $q_{2}^{\psi}=0$ was decided. This set will be implemented in the AFS controller (17) for 
TABLE III

Profiles Of Driver Lane Change Target Paths

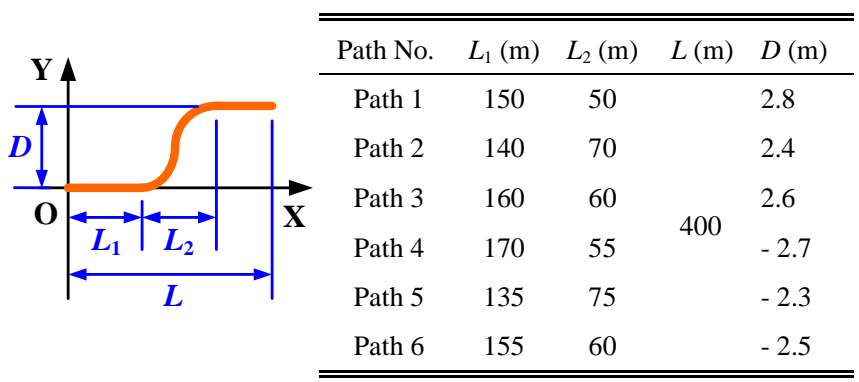

measuring driver steering behavior in the experiment. On this basis, another two weight sets were derived to extend the driver behavior measurement. All the three weight sets are:

AFS Weight Set 1: $q_{2}^{y}=0.6 \mathrm{e}-3, q_{2}^{y_{\mathrm{int}}}=0$, and $q_{2}^{\psi}=0$.

AFS Weight Set 2: $q_{2}^{y}=1.2 \mathrm{e}-3, q_{2}^{y_{\text {int }}}=0$, and $q_{2}^{\prime \prime}=0$.

AFS Weight Set 3: $q_{2}^{y}=0.6 \mathrm{e}-3, q_{2}^{y_{\text {int }}}=1.5 \mathrm{e}-8$, and $q_{2}^{\psi}=0$.

Note that AFS Weight Set 2 is the one determined directly from the trials. AFS Weight Set 1 was derived from Weight Set 2 by reducing the value of $q_{2}^{y}$ to a half. This allows the driver to override the AFS control more easily. AFS Weight Set 3 was derived from Weight Set 1 by further penalizing vehicle lateral displacement integral error using $q_{2}^{y_{\text {int }}}=1.5 \mathrm{e}-8$. This increases the difficulty for the driver to override the AFS control.

At this point, two remarks are made. First, in the trials the AFS controller was programmed to start lane change at random positions to avoid a subject memorizing the location where the AFS started to intervene. As a subject was exposed to the AFS control repeatedly, they may be more prepared to counter AFS than in real driving. This is thought a limitation. In real driving, a driver may be less capable of overriding the AFS control with $q_{2}^{y}=1.2 \mathrm{e}-3$. As this paper aims to evaluate driver model (12) for representing human driver steering behavior, it is thought that $q_{2}^{y}=1.2 \mathrm{e}-3$ can still give valid research results.

Second, the tire cornering stiffness of vehicle model (3) were assumed constant in the trials. But in reality, it will decrease as brake force increases, due to tire force saturation. Hence, the AFS control with the same path-error weights will be harder for drivers to override in real driving. With some priori knowledge of the experiment results (to be presented in Section V), all the six subjects listed in Table II were found able to override the AFS control with $q_{2}^{y}=1.2 \mathrm{e}-3$. Therefore, this value is thought acceptable, and less likely to weaken the soundness of the model evaluation work, which will be described in Section V.

Simulation results of the path-following performance of AFS controller (17) with the three weight sets were illustrated in Fig. 5. The AFS target path is the same as the one shown in Fig. 3. The lane change begins at a longitudinal position of $120 \mathrm{~m}$. No steering action is applied by the driver. The AFS steering angles under the three weight sets are shown in the right plot in solid, dashed and dotted lines, respectively. They are very similar to each other. Therefore, the vehicle lateral displacement resulted from the three weight sets are close, as shown in the left plot. It can be seen that all the three AFS weight sets result in decent path-following performance, with vehicle lateral displacement

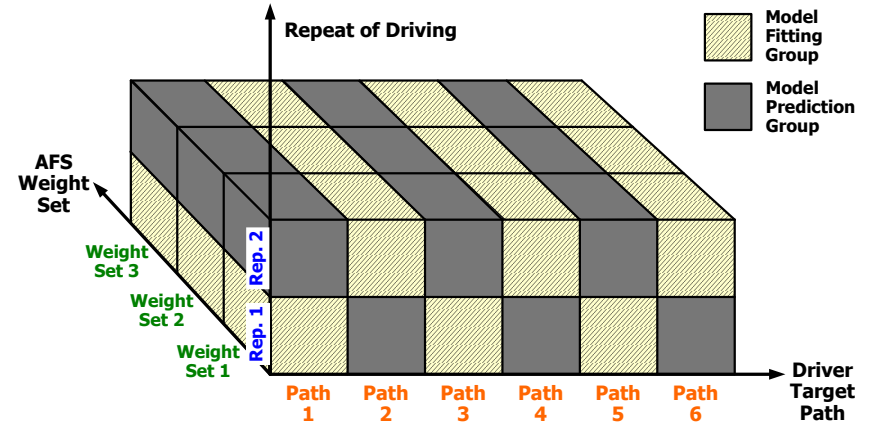

Fig. 7. Experiment data sets for model identification and validation.

overshoot smaller than $0.5 \mathrm{~m}$.

It was stated that AFS controller (17) adopts a design concept of compensating for driver steering angle to mitigate possible adverse impact due to driver error. To this end, the three AFS weight sets were also inspected when driver applies disturbance steering control. The disturbance is simulated using a pseudorandom steering angle history generated using Fourier series. Vehicle lateral motion resulted from driver-AFS interaction are shown in Fig. 6. It can be seen that the three AFS weight sets again resulted in very similar AFS angles. In each case, the AFS controller opposed the driver and enabled the vehicle to roughly follow the AFS lane-change target path. The AFS Weight Set 2 (dashed lines) that has the largest $q_{2}^{y}$ resulted in the least pathfollowing error. Compared to AFS Weight Set 1 (solid lines), Weight Set 3 that involves integral error weight $q_{2}^{y_{\text {int }}}=1.5 \mathrm{e}-8$ reduced vehicle path-tracking error. Although the three AFS weight sets lead to little significant difference in vehicle lateral motion, they did cause distinct driver steering reaction in the experiment. Detailed discussions will be given in section V.

\section{Experiment Driving Scenario}

On completing the trial, the experiment for measuring driver subjects' steering behavior was started. The driving scenario used in the experiment is distinct from that in the trials. In the experiment, vehicle model (3) was programmed to travel at a constant longitudinal speed of $U=20 \mathrm{~m} / \mathrm{s}$, and AFS controller (17) was designed to conduct active lane keeping control so as to keep the vehicle staying in the lane. In contrast, each subject in Table II was asked to conduct lane-change maneuvers. In this scenario, the AFS controller' target path is a straight line ahead, which differs greatly from the driver's lane-change target path. This may cause the AFS and the driver to compete for vehicle directional control in such a way that the driver tries to steer the vehicle away from the lane while the AFS tries to steer back to the lane. Such a scenario may occur when the AFS lane keeping control fails to recognize a lane drop due to road works, as described in [36], or when the AFS faces a 'social dilemma' between colliding to a wall or a pedestrian, as studied in [37].

Following designing the experiment driving scenario, six mild lane-change target paths were constructed for the driver, as shown in Table III. Each subject was asked to control the vehicle to follow these lane-change paths, in the presence of AFS lane keeping control that use the three AFS weight sets described above. In other words, each subject faced 18 different 


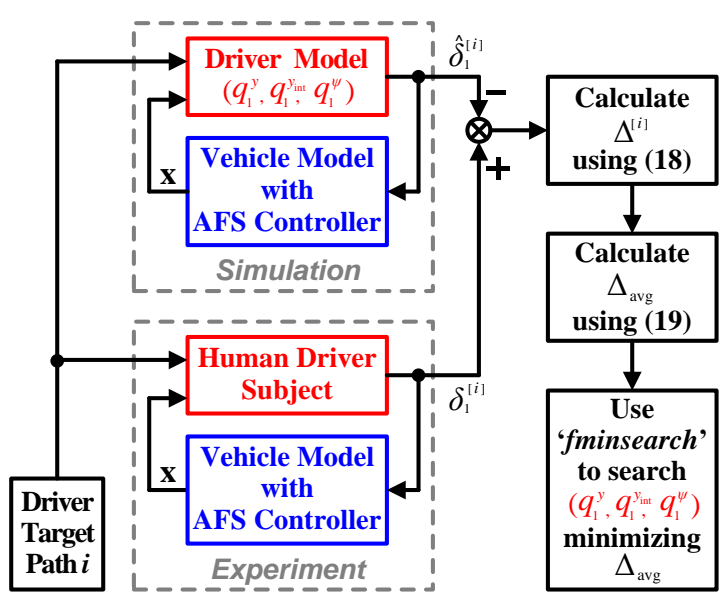

Fig. 8. Diagram of 'indirect' method for identifying driver model parameter set $\left(q_{1}^{y}, q_{1}^{y_{\text {int }}}, q_{1}^{\psi}\right)$.

driver-AFS interaction events (3 AFS weight sets x 6 driver lane-change target paths). Subjects' steering angles measured over these events will be used for driver model evaluation.

To hinder a test subject from memorizing when the AFS lane keeping happens in an experiment, three forms of disturbance events were designed: (i) step AFS angle disturbance, (ii) ramp AFS angle disturbance, and (iii) cross-wind disturbance. More details on the design of the disturbance event were given in [34]. In the experiment, the 18 driver-AFS interaction events were mixed with the disturbing events in a random order.

\section{E. Experiment Protocol and Data Acquisition}

During the experiment, each of the six subjects was asked to complete two repeats of the 18 driver-AFS interaction events. The subjects were neither told the existence of AFS active lane keeping nor the nature of the research. They were simply asked to follow their target paths while advised the existence of some disruption. Test subject's steering wheel angle, AFS angle, and vehicle states were measured and recorded. The other signals described in Fig. 2 (a) were not recorded.

On completing two repeats of the 18 driver-AFS interaction events, each subject gave 36 data sets representing his or her steering control in response to AFS lane keeping control. These 36 data sets were depicted as cubes in Fig. 7, and divided into two groups. One group is named 'Model Fitting Group'. Driver Nash steering strategy (12) will be fitted to the data belonging to this group to identify the key parameters involved in (12). The other group is named "Model Prediction Group". Data of this group will be used to validate the model fitting outcomes.

The time taken for a subject to complete two repeats of the 18 driver-AFS interaction events along with the disturbance events is about 40 minutes. To avoid driving fatigue, each subject was asked to take a rest after 20 minutes' driving. The subjects were observed busy reacting to the events throughout the experiment. Interviews revealed that they could hardly distinguish the effects of AFS lane keeping control from the disturbances.

\section{DRIVER MODEL FITTING}

As stated previously, this paper aims to evaluate if the game-

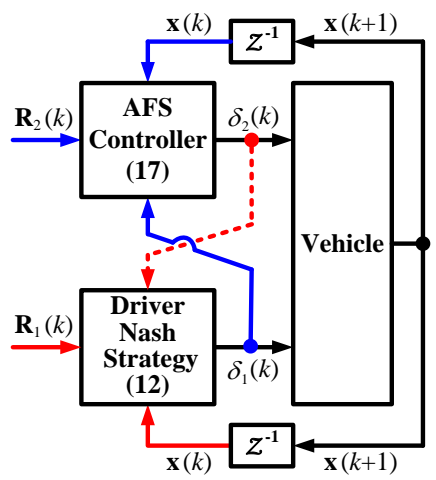

(a)

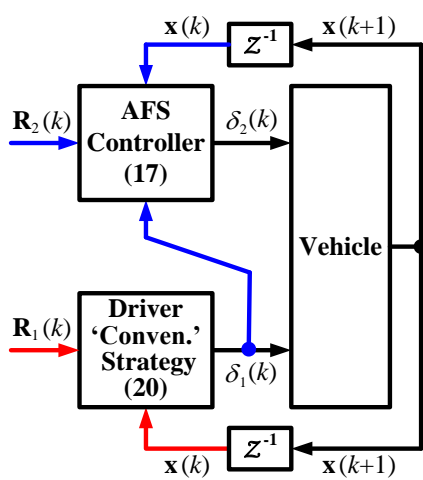

(b)
Fig. 9. Schemes of driver-AFS steering interaction in two driver model fitting tasks. (a) Model fitting task 1, where game-theoretic driver model is in use. (b) Model fitting task 2, where 'conventional' driver model is in use.

theoretic driver model (12) is advantageous over an optimalcontrol-theoretic model (21) (to be described in Section IV) for representing individual human drivers' steering behavior. To this end, (12) and (21) were respectively fitted to the steering angles measured from each of the six subjects. Driver model parameters allowing a best fit of the model output to measured steering angles were identified. The method and procedure for model fitting are explained in this section.

\section{A. Model Fitting Method}

The steering angle generated by driver Nash strategy (12) is dominated by driver preview horizon $N_{1}$ and path-error weights $q_{1}^{y}, q_{1}^{y_{\text {it }}}$ and $q_{1}^{\psi}$. Fitting (12) to measured driver steering angle therefore involves identifying an appropriate set $\left(N_{1}, q_{1}^{y}\right.$, $\left.q_{1}^{y_{\text {int }}}, q_{1}^{\psi}\right)$ that allows the best fit. To enhance identification efficiency, driver preview horizon $N_{1}$ is fixed at 200 steps. This corresponds to a preview time of $2.0 \mathrm{~s}$ (at sampling time $T=$ $0.01 \mathrm{~s}$ ). This value is chosen according to the findings reported in [12] and [13]: it was found that in path-following scenarios, a driver steering control model using a preview time of $2.0 \mathrm{~s}$ generally resulted in better representation of measured driver steering behavior than using other preview times, ranging from $0.5 \mathrm{~s}$ to $1.75 \mathrm{~s}$. Following fixing $N_{1}=200$, the driver model parameter set to be identified reduces to $\left(q_{1}^{y}, q_{1}^{y_{\mathrm{int}}}, q_{1}^{\psi}\right)$.

The 'indirect' method proposed by Ljung [38] is adopted to determine a driver model parameter set $\left(q_{1}^{y}, q_{1}^{y_{\text {int }}}, q_{1}^{\psi}\right)$ that allows a best fit of (12) to measured driver steering angles. Fig. 8 schematically describes the procedure. The 'Experiment' area enclosed by dashed lines displays what happened in the experiment: a subject was asked to control vehicle model (3) to perform mild lane-change maneuvers following his/her target path $i(i=1,2, \ldots, 6$, as shown in Table III). Meanwhile, AFS controller (17) performed active lane keeping control to keep the vehicle staying in its original lane. The AFS controller was parameterized by one of the three AFS Weight Sets described previously. $\delta_{1}^{[i]}$ denotes the driver steering angle measured under target path $i$. The 'Simulation' area involves the driver model of interest running in closed loop with vehicle model (3) 

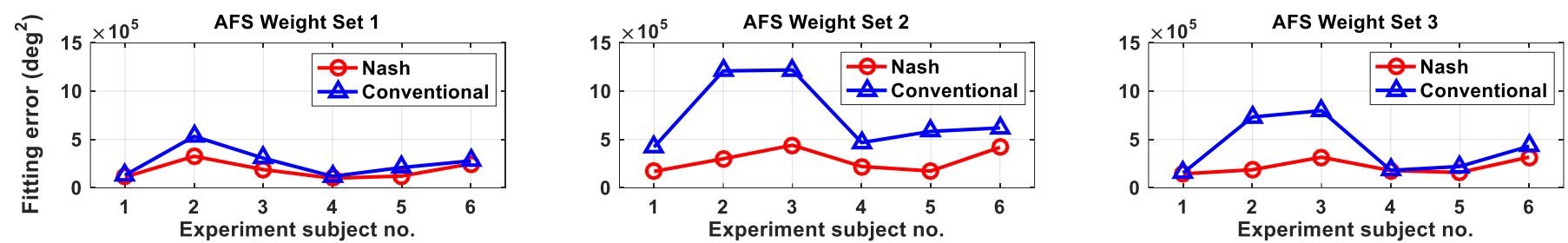

Fig. 10. Model fitting errors resulted from fitting game-theoretic driver model (circle marker), and 'conventional' driver model (triangle marker) to steering angles measured from six driver subjects, under three designated AFS weight sets.

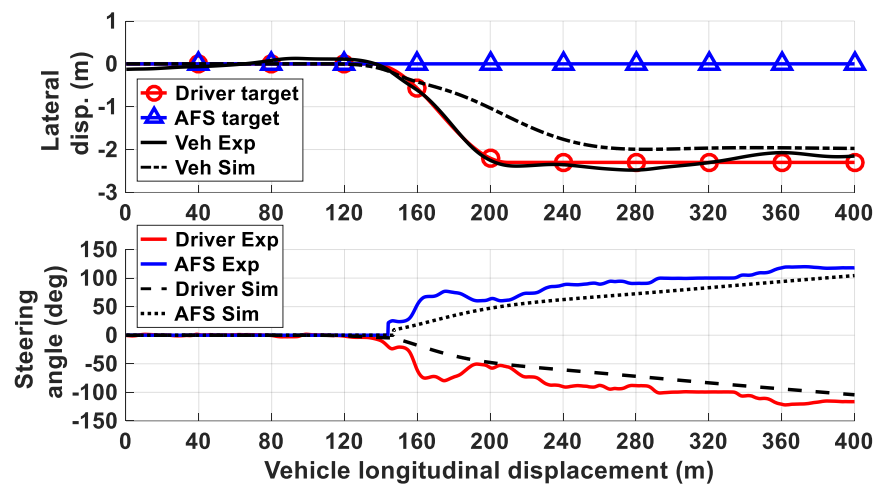

(a)

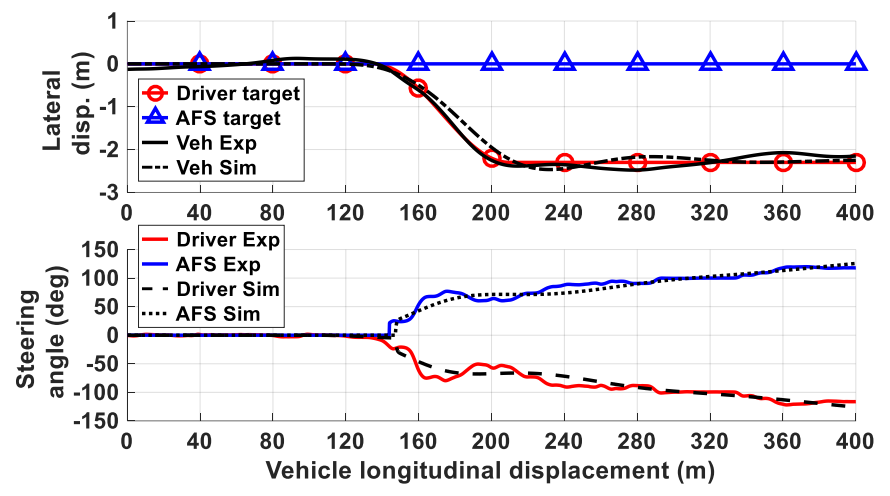

(b)

Fig. 11. Experimental results (denoted as 'Exp') and simulated results (denoted as 'Sim') of Subject 2 under AFS Weight Set 3 and driver lane-change target path 5. (a) Best fit of 'conventional' driver model to experimental data; (b) Best fit of game-theoretic driver model to experimental data.

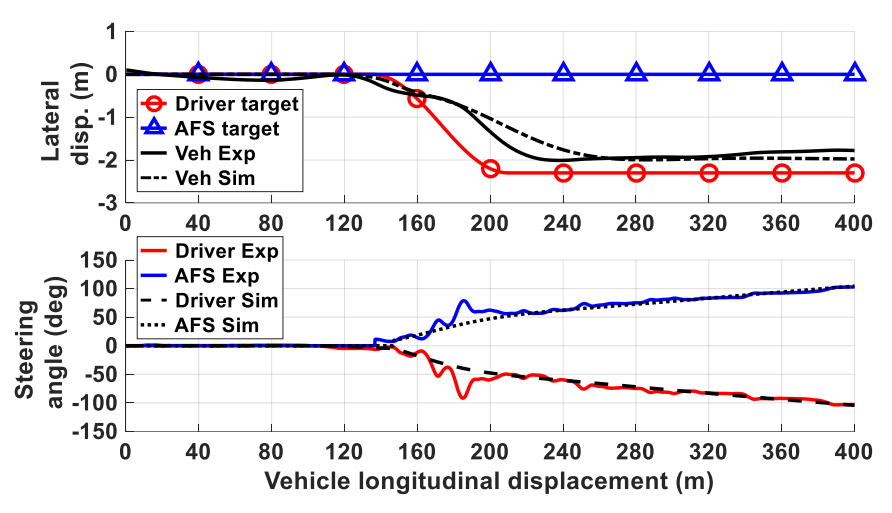

(a)

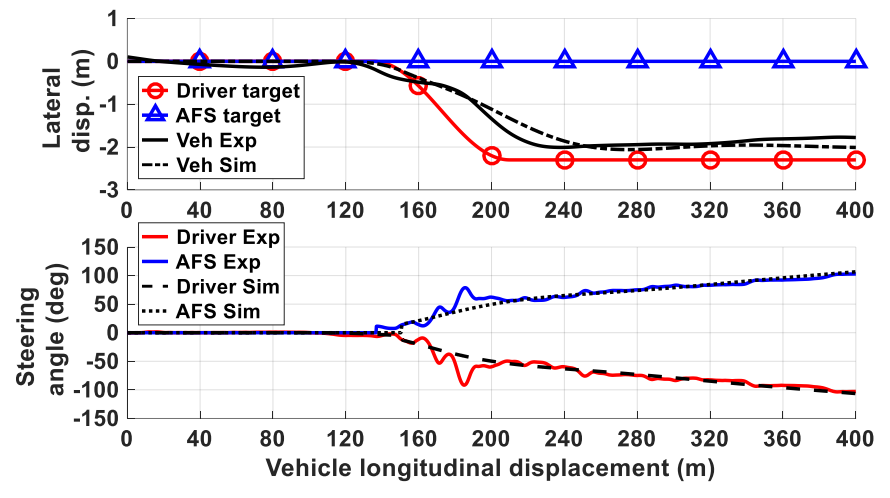

(b)

Fig. 12. Experimental results (denoted as 'Exp') and simulated results (denoted as 'Sim') of Subject 4 under AFS Weight Set 3 and driver lane-change target path 5. (a) Best fit of 'conventional' driver model to experimental data; (b) Best fit of game-theoretic driver model to experimental data.

and AFS controller (17). The driver model is fed with the same target path as that for the 'Experiment' area. $\hat{\delta}_{1}^{[i]}$ denotes the simulated driver steering angle under target path $i . \hat{\delta}_{1}^{[i]}$ is dominated by driver path-error weight set $\left(q_{1}^{y}, q_{1}^{y_{\text {iti }}}, q_{1}^{\psi}\right)$.

The model fitting error associated with a driver subject under target path $i$ is defined as $\Delta^{[i]}$ calculated using (18):

$$
\Delta^{[i]}=\sum_{k=k_{\mathrm{S}}}^{k_{\mathrm{E}}}\left[\delta_{1}^{[i]}(k)-\hat{\delta}_{1}^{[i]}(k)\right]^{2}
$$

It can be seen that $\Delta^{[i]}$ is essentially the sum of squared error between measured and simulated driver steering angles. $k_{\mathrm{S}}$ and $k_{\mathrm{E}}$ denote the start and end time steps of driver target path $i$. As the length of a driver target path $L$ is $400 \mathrm{~m}$ (see Table III), there are 2000 steps between $k_{\mathrm{S}}$ and $k_{\mathrm{E}}$. This is calculated using $L=$ $400 \mathrm{~m}$, vehicle speed $U=20 \mathrm{~m} / \mathrm{s}$, and sample time $T=0.01 \mathrm{~s}$.

Continuing to follow Fig. 8, the model fitting errors obtained from the six driver lane-change target paths are then averaged:

$$
\Delta_{\mathrm{avg}}=\frac{1}{6} \cdot \sum_{i=1}^{6} \Delta^{[i]}
$$

Finally, the Matlab function 'fminsearch' is used to search for a driver control weight set $\left(q_{1}^{y}, q_{1}^{y_{\text {int }}}, q_{1}^{\psi}\right)$ that minimizes the averaged model fitting error $\Delta_{\text {avg }}$ associated with a specific subject and a specific AFS weight set. The 'fminsearch' adopts 
TABLE IV

P-VALUES OF ONE-TAILED HYPOTHESIS TEST

\begin{tabular}{ccccccc}
\hline \hline & \multicolumn{6}{c}{ Subject No. } \\
\cline { 2 - 7 } & 1 & 2 & 3 & 4 & 5 & 6 \\
\hline$p$-value & 0.0885 & 0.0002 & 0.0003 & 0.0083 & 0.0007 & 0.0226 \\
\hline \hline
\end{tabular}

the Nelder-Mead simplex search algorithm described in [39]. This algorithm involves an iterative process: at each searching step a new driver weight set is generated based on the simplex of the weight set generated at the last step. The averaged model fitting error $\Delta_{\text {avg }}$ resulted from the current step is compared to that from the last step. The searching process stops when a minimum $\Delta_{\text {arg }}$ is found. The initial values of $q_{1}^{y}, q_{1}^{y_{\text {itt }}}$ and $q_{1}^{\psi}$ were all set 1e-8. The maximum iteration number was set 1000 . Preliminary tests showed that the driver weights identified via 'fminsearch' were insensitive to their initial values, and the iteration number was sufficiently large to reach a convergence.

\section{B. Model Fitting Tasks}

Two model fitting tasks, namely tasks 1 and 2 were carried out. Task 1 involves fitting the game-theoretic driver model (12) to each subject's measured steering behavior. Task 2 involves fitting the optimal-control-theoretic driver model (21) (to be described later in this subsection) to the same measured data.

\section{1) Model fitting task 1}

This task involves fitting the driver Nash steering strategy, or in other words, the game-theoretic driver model (12) to the steering angles measured from each of the six subjects listed in Table II. The outcome is a specific driver model weight set $\left(q_{1}^{y}\right.$, $\left.q_{1}^{y_{\text {it }}}, q_{1}^{\psi}\right)$ allowing the best fit. The method and procedure for this task was illustrated in Fig. 8. As stated in Section II, driver model (12) involves assuming that the driver determines his/her steering angle $\delta_{1}(k)$ using vehicle state $\mathbf{x}(k)$ and driver target path $\mathbf{R}_{1}(k)$, while compensating for AFS angle $\delta_{2}(k)$. Fig. 9 (a) outlines such driver-AFS interaction. Note that the scheme shown in Fig. 9 (a) is identical to the one shown in Fig. 1.

\section{2) Model fitting task 2}

This task is concerned with fitting a 'conventional' optimalcontrol-theoretic driver model to the steering angles measured from the six subjects. This task serves as a comparator to task 1 . The application of optimal control theory to modeling driver steering control was first reported in [40]. Since then, optimalcontrol-theoretic driver models have been widely used to model driver steering behavior exhibited in various driving scenarios. While a review on this point was given in Section I, it is worth noting that different forms of optimal-control-theoretic driver models, such as those reported in [11-13] and [40] were derived following the same principle. This involves a key assumption that the driver determines his/her steering angle $\delta_{1}(k)$ using vehicle state $\mathbf{x}(k)$ and driver target path $\mathbf{R}_{1}(k)$ only. In other words, the driver model was not equipped with the feature of compensating for control actions from vehicle automation.

A 'conventional' optimal-control-theoretic driver model can be derived following a two-stage procedure. First, the driver's
TABLE V

VALUES OF FRACTION OF VARIANCE UNEXPLAINED (FVU)

\begin{tabular}{cccc}
\hline \hline \multirow{2}{*}{$\begin{array}{c}\text { Subject } \\
\text { No. }\end{array}$} & \multicolumn{2}{c}{ FVU: 'conventional' model vs. game theoretic model } \\
\cline { 2 - 4 } & AFS Weight Set 1 & AFS Weight Set 2 & AFS Weight Set 3 \\
\hline 1 & 0.095 vs. 0.081 & 0.082 vs. 0.033 & 0.043 vs. 0.040 \\
2 & 0.299 vs. 0.183 & 0.196 vs. 0.049 & 0.149 vs. 0.038 \\
3 & 0.166 vs. 0.103 & 0.178 vs. 0.064 & 0.151 vs. 0.060 \\
4 & 0.099 vs. 0.083 & 0.102 vs. 0.048 & 0.054 vs. 0.054 \\
5 & 0.120 vs. 0.071 & 0.098 vs. 0.029 & 0.059 vs. 0.043 \\
6 & 0.182 vs. 0.163 & 0.116 vs. 0.078 & 0.100 vs. 0.074 \\
\hline \hline
\end{tabular}

prediction equation is constructed as:

$$
\mathbf{Z}_{1}(k)=\boldsymbol{\Psi}_{1} \mathbf{x}(k)+\boldsymbol{\Theta}_{1} \delta_{1}(k)
$$

Compared to the driver's prediction equation (5) for the gametheoretic driver model, (20) does not have the term associated with AFS angle $\delta_{2}(k)$. This complies with the assumption that the driver does not compensate for AFS control. Second, driver cost function (6) is minimized subject to (20). This results in the optimal-control-theoretic driver steering strategy, or in other words, the 'conventional' driver model (21):

$$
\delta_{1}(k)=\tilde{\mathbf{G}}_{1}\left\{\begin{array}{c}
\mathbf{x}(k) \\
\mathbf{R}_{1}(k)
\end{array}\right\}
$$

$\tilde{\mathbf{G}}_{1}$ denotes the gain array. By comparing (21) to the gametheoretic driver model (12), it can be seen that (21) does not incorporate AFS angle $\delta_{2}(k)$. Fig. 9 (b) illustrates the scheme of driver model (21). Compared to Fig. 9 (a), Fig. 9 (b) does not involve feeding the AFS angle $\delta_{2}(k)$ to the driver. As cost function (6) comprises driver path-error weights $q_{1}^{y}, q_{1}^{y_{\text {iit }}}$ and $q_{1}^{\psi}$, the steering angle determined by the 'conventional' driver model (20) is thereby dominated by these weights. Hence, the model fitting task 2 is centered on identifying a $\left(q_{1}^{y}, q_{1}^{y_{\text {iit }}}, q_{1}^{\psi}\right)$ set that allows a best fit of driver model (21) to measured driver steering behavior. This task can be carried out by following the method and procedure described in Fig. 8.

It was observed that with a computer having an Intel Core i7 3.50 GHz CUP and 16.0 GB RAM, the time taken to identify a driver control weight set for a subject under a specific AFS weight set was less than 2 minutes on average.

\section{RESULTS AND DISCUSSION}

\section{A. Model Fitting Results}

In Section VI, the 'conventional' driver model (21) was introduced as a comparator to the game-theoretic model (12), and the two models were fitted to experimental measurements, respectively. The averaged model fitting errors $\Delta_{\text {avg }}$ as per (18) resulted from the model fitting are shown in Fig. 10. It can be seen from Fig. 10 that for all subjects, the game-theoretic driver model (12) yields smaller fitting errors than the 'conventional' model (21) under all three AFS weight sets. In other words, the game-theoretic model seems to give closer representation of subjects' steering behavior. To verify whether the model fitting 

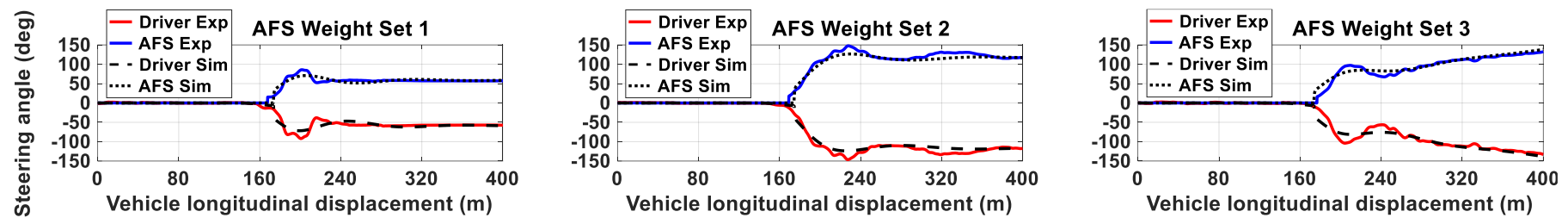

Fig. 13. Experimental results (denoted as 'Exp') and driver game-theoretic model simulated results (denoted as 'Sim') of Subject 2 along driver lane-change target path 4 , under three designated AFS weight sets.
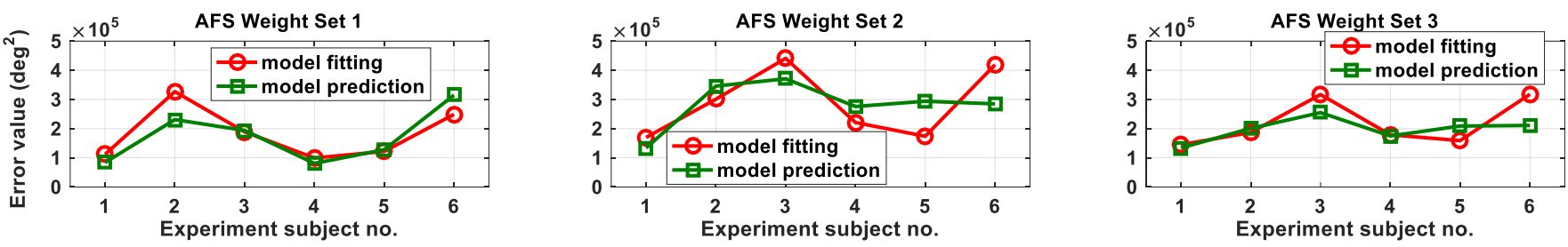

Fig. 14. Comparison between subjects' model fitting errors (circle marker) and model prediction errors (square marker), with game-theoretic driver model in use.

errors resulted from the game-theoretic model, as shown in Fig. 10 are significantly smaller than those from the 'conventional' model, a one-tailed hypothesis test is taken. The null hypothesis $\boldsymbol{H}_{\mathbf{0}}$ and the alternative one $\boldsymbol{H}_{1}$ are stated respectively as:

$\boldsymbol{H}_{\mathbf{0}}$ : the model fitting errors given by game-theoretic model (12) are no smaller than those by the 'conventional' model (21).

$\boldsymbol{H}_{1}$ : the model fitting errors given by game-theoretic model (12) are smaller than those by the 'conventional' model (21).

Note that the hypothesis test involves treating each subject as an individual, rather than viewing all of them as a population. As described in Fig. 7, each subject's 'Model Fitting Group' consists of 18 data sets ( 6 target paths x 3 AFS weights). Hence, for each subject there exist a sample of 18 model fitting errors associated with the game-theoretic model (12), and another sample of 18 error values associated with the 'conventional' model (21). This hypothesis test is for evaluating if the fitting errors in the game-theoretic model sample are statistically significantly smaller than those in the 'conventional' model sample. To this end, the Wilcoxon matched-pairs signed-rank test [41] was adopted. The test method is nonparametric. It does not require the assumption that the samples should be from a parametric family of distribution. The test in its two-tailed form was used by Inga et al. [42] to evaluate the significance of the difference in cost function parameters of two driver models.

The $p$-values resulted from testing the hypothesis $\boldsymbol{H}_{\mathbf{0}}$ for each subject are shown in Table IV. With a significance level of $\alpha=$ 0.01 , hypothesis $\boldsymbol{H}_{\mathbf{0}}$ in Subjects 2, 3 and 5's cases is rejected as their corresponding $p$-values are smaller than $\alpha / 2$. In contrast, $\boldsymbol{H}_{\mathbf{0}}$ in Subjects 1, 4 and 6's cases cannot be rejected. This result suggests that the game-theoretic model (12) lead to statistically significantly smaller model fitting errors than the 'conventional' model (21) for Subjects 2, 3 and 5, but not for Subjects 1, 4 or 6 . In other words, the game-theoretic model (12) is statistically advantageous over the 'conventional' driver model (21) in representing Subject 2's, 3's and 5's steering behavior.

The performance of driver models (12) and (21) in terms of how well they fit the measured driver behavior are quantified using an index called fraction of variance unexplained (FVU) [41]. For each subject, the FVU given by the game-theoretic driver mode (12) and that by the 'conventional' model (21) are determined under each of the three AFS Weight Sets using:

$$
\mathrm{FVU}=\frac{\text { variance of model fitting error }}{\text { variance of measured driver steer angle }}
$$

Table $\mathrm{V}$ shows the FVU values given by the two driver models. The FVU by definition varies between 0 and 1 . A value closer to 0 indicates a better fit to the experiment data. It can be seen that Subjects 2's, 3's and 5's FVUs given by the game-theoretic model (12) are markedly smaller than by the 'conventional' model (21). This finding is in accordance with that from the hypothesis test. Of particular note is that under AFS Weight Set 3, Subject 4's FVUs given by the two driver models are both 0.054 . Such a small FVU implies that the two models fit the experiment data equivalently well. In contrast, Subject 2's FVU given by the game-theoretic model is 0.038 , which is $75 \%$ smaller than the 0.149 by the conventional model. This means that the game-theoretic model fits the measured data fairly well while the 'conventional' model is poor in this case.

As stated above, Subject 2 is among the three subjects whose steering behavior was better represented by the game-theoretic model (12), compared to the 'conventional' model (21). Fig. 11 shows how the two models perform in representing Subject 2's steering behavior under AFS Weight Set 3. The experimental data in Fig. 11 (denoted as 'Exp') suggest that Subject 2 was able to override AFS lane keeping control and get the vehicle to follow his lane-change target path. When 'conventional' model (20) was used to represent Subject 4's control behavior, poor agreement between simulated and measured driver steering angle was seen, as per Fig. 11 (a): the simulated driver steering angles fail to track the experimental measurement; an offset of 15 deg exists. Such poor agreement in turn causes the simulated vehicle motion to deviate from the measurement. In contrast, when game-theoretic model (11) was employed, much better agreement was seen, as illustrated Fig. 11 (b): the simulated driver angles capture the measured ones well. This enables the 
simulated vehicle motion to track the measurement well.

Subject 4 is one of the three subjects whose hypothesis tests do not show a significance difference between using the gametheoretic model and the 'conventional' model for representing their steering behavior. Fig. 12 illustrates how the two models perform in Subject 4's case under AFS Weight Set 3. Fig. 12 (a) displays the simulated results from fitting the 'conventional' model (21) to experimental data while (b) shows those from fitting the game-theoretic model (12). It can be found that the measured vehicle lateral displacement does not firmly track the driver's lane-change target path - there exists some steady-state path-following error. This suggests that Subject 4 was not good at suppressing the AFS lane keeping control. The simulated results from the game-theoretic model (12) shown in Fig. 12 (b) are closely similar to those from the 'conventional' model (21) shown in Fig. 12 (a). Simulation results from both models fit Subject 4's measured steering angle and vehicle motion well.

Fig. 13 shows Subject 2's experimental results and the gametheoretic model's simulated results along driver lane-change target path 4 under all the three AFS weight sets. It can be seen that the simulated steering angles fit the experimental data well. The three subplots respectively correspond to FVU values of $0.183,0.049$ and 0.038 shown in Table V. As a result of raising AFS path-error weight $q_{2}^{y}$ from 0.6e-3 (AFS Weight Set 1) to 1.2e-3 (AFS Weight Set 2), Subject 2 doubled steering angle magnitude. By adding AFS lateral displacement error integral weight $q_{2}^{y_{\text {itt }}}=1.5 \mathrm{e}-8$ (AFS Weight Set 3), Subject 2 had to keep increasing steering angle to oppose AFS control. Due to space limitation, subjects' identified path-error weights $q_{1}^{y}, q_{1}^{y_{\text {iit }}}, q_{1}^{\prime \prime}$ are not presented in the present paper but can be found in [34].

\section{B. Model Prediction Results}

The simulated steering angle and vehicle motion histories presented above were obtained by fitting the two driver models to the experimental data belong to the 'Model Fitting Group', as described in Fig. 7. To further examine if the identified gametheoretic driver model is capable of predicting driver steering behavior, a new index named model prediction error $\Delta_{\text {avg }}^{\text {pre }}$ is defined. Determination of $\Delta_{\text {avg }}^{\text {pre }}$ follows a similar way to that of the model fitting error $\Delta_{\text {avg }}$, as defined by (18) and (19). The key difference lies in the experimental data used: model fitting error $\Delta_{\text {avg }}$ is calculated using the experimental data belonging to the 'Model Fitting Group' shown in Fig. 7, while model prediction error $\Delta_{\text {avg }}^{\text {pre }}$ is computed using the data belonging to the 'Model Prediction Group'. Fig. 14 presents the comparison between subjects' $\Delta_{\text {avg }}^{\text {pre }}$ and $\Delta_{\text {avg }}$. The $\Delta_{\text {avg }}$ presented in Fig. 14 (circle marker) are copied from those shown in Fig. 10, with the game-theoretic model (12) in use. It can be found that Subjects 1 's to 5 's model prediction errors $\Delta_{\text {avg }}^{\text {pre }}$ (square marker) are close to their model fitting errors $\Delta_{\text {avg }}$. This suggests that the game-theoretic model (12) identified from these five subjects using their 'Model Fitting Group' data is able to predict their steering behavior exhibited in the 'Model Prediction Group'. This in turn demonstrates the predictivity of the game-theoretic driver model (12) under the driving scenario concerned. For Subject 6, obvious differences between his model prediction error $\Delta_{\text {avg }}^{\text {pre }}$ and model fitting error $\Delta_{\text {avg }}$ were seen. This was found due to the oscillations involved in the subject's steering behavior. Subject 6 found it difficult to stabilize the vehicle when his interacting with the AFS lane keeping control: he turned the steering wheel very frequently to control the vehicle. As the game-theoretic driver model (12) studied in this paper does not account for a driver's arm neuromuscular dynamics, Subject 6's oscillatory steering behavior could not be captured. Due to space limit, discussion on this issue was not extended in the present paper, but more details were provided in [34].

\section{CONCLUSION}

This paper centers the evaluation of a game-theoretic driver steering control model for its advantage over optimal-controltheoretic driver model in representing human drivers' steering behavior in response to vehicle automated steering control in a lane keeping scenario. A driving simulator experiment with six driver subjects were conducted, and their steering angles were measured. Key parameters of the game-theoretic driver model and its comparator were identified via a system identification method using the measurement. Statistical analyses were made to evaluate the performance of the driver models. The gametheoretic driver model was found significantly better than its optimal-control-theoretic comparator in representing three of the six subjects' measured steering behavior. For the other three subjects, the two driver models were found equivalently well at representing the subjects' measured steering behavior.

The work has some limitations: i) the number of subjects is not sufficient to represent the steering behavior of the driver population; ii) the subjects in the experiment may be aware of the intervention of the automated steering, and thus may be more prepared than in real driving; and iii) the capability of the game-theoretic driver model for predicting drivers' behavior shown in different driving scenarios was not fully investigated. Despite the limitations, the findings can suggest some potential advantage of the game-theoretic model in representing drivers' steering behavior when their objectives in vehicle control differ from the automated controller's. In the future, evaluation of the game-theoretic model using subjects from a wider demographic range in the presence of a wider range of automated steering technology and driving scenarios will be conducted.

\section{REFERENCES}

[1] D. Soudbakhsh and A. Eskandarian, "Steering control collision avoidance system and verification through subject study," IET Intell. Transp. Syst., vol. 9, no. 10, pp. 907-915, Dec. 2015.

[2] T. Dang, J. Desens, U. Franke, D. Gavrila, L. Schäers, and W. Ziegler, "Steering and evasion assist," in Handbook of Intelligent Vehicles, A. Eskandarian, Ed. London, U.K.: Springer-Verlag, 2012, pp. 759-782.

[3] S. J. Anderson, S. C. Peters, T. E. Pilutti, and K. Iagnemma, "An optimalcontrol-based framework for trajectory planning, threat assessment, and semi-autonomous control of passenger vehicles in hazard avoidance scenarios," Int. J. Veh. Auton. Syst., vol. 8, nos. 2/3/4, pp. 190-216, 2010.

[4] R. Isermann, R. Mannale, and K. Schmitt, "Collision-avoidance systems PRORETA: situation analysis and intervention control," J. Control. Eng. Pract., vol. 20, no. 11, pp. 1236-1246, Nov. 2012.

[5] V. Turri, A. Carvalho, H. E. Tseng, K. Johansson, and F. Borrelli, "Linear model predictive control for lane keeping and obstacle avoidance on low curvature roads," in Proc. IEEE Int. Transp. Syst. Conf., Hague, The Netherlands, 2013.

[6] E. Bender, K. Landau, and R. Bruder, "Driver reactions in response to automatic obstacle avoiding manoeuvres," in Proc. World Congr. Ergonom., Maastricht, The Netherlands, 2006.

[7] M. Itoh, T. Horikome, and T. Inagaki, "Effectiveness and driver 
acceptance of a semi-autonomous forward obstacle collision avoidance system," in Proc. Human Factors and Ergonom. Soc. Annu Meeting, San Francisco, CA, U.S.A., 2010.

[8] M. Irmscher, T. Jürgensohn, and H. Willumeit, "Driver models in vehicle development," Veh. Syst. Dyn., vol. 33, sup. 1, pp. 83-93, 1999.

[9] M. Plöchl and J. Edelmann, "Driver models in automobile dynamics application," Veh. Syst. Dyn., vol. 45, no. 7-8, pp. 699-741, Jul. 2007.

[10] G. Markkula, "Driver behavior models for evaluating automotive active safety," Ph.D. dissertation, Chalmers University of Technology, Sweden, 2015.

[11] A. Y. Ungoren and H. Peng, "An adaptive lateral preview driver model," Veh. Syst. Dyn., vol. 43, no. 4, pp. 245-259, Apr. 2005.

[12] S. D. Keen and D. J. Cole, "Bias-free identification of a linear modelpredictive steering controller from measured driver steering behavior," IEEE Trans. Syst., Man, Cybern. B, Cybern., vol. 42, no. 2, pp. 434-443, 2012.

[13] A. M. C. Odhams and D. J. Cole, "Identification of the steering control behaviour of five test subjects following a randomly curving path in a driving simulator", Int. J. Veh. Auton. Syst. vol. 12, no. 1, pp. 44-64, 2014.

[14] N. Winkler, L. Drugge, A. S. Trigell, and G. Efraimsson, "Coupling aerodynamics to vehicle dynamics in transient crosswinds including driver model," Computers and Fluids, vol. 138, pp. 26-34, Oct. 2016.

[15] D. J. Cole, "A path-following driver-vehicle model with neuromuscular dynamics, including measured and simulated responses to a step in steering angle overlay," Veh. Syst. Dyn., vol. 50, no. 4, pp. 573-596, Apr. 2012.

[16] D. A. Braun, P. A. Ortega, and D. M. Wolpert, "Nash equilibria in multiagent motor interactions," PLOS Comput. Biol., vol. 5, no. 8, pp. 1-8, 2009.

[17] G. Schmid and D. A. Braun, "Human group coordination in a sensorimotor task with neuron-like decision-making," Nature: Scientific Rep., vol. 10, no. 8226, 2020.

[18] D. A. Braun, P. A. Ortega, and D. M. Wolpert, "Motor coordination: when two have to act as one," Exp. Brain Res., vol. 211, pp. 631-641, 2011.

[19] F. Leibfried, J. Grau-Moya, and D. A. Braun, "Signaling equilibria in sensorimotor interactions," Cognition, vol. 141, pp. 73-86, 2015.

[20] V. T. Chackochan and V. Sanguineti, "Modeling collaborative strategies in physical human-human interaction," in Converging Clinical and Eng. Research on Neurorehabilitation. Springer, vol. 15, pp. 253-258, 2017.

[21] Y. Li, G. Carboni, F. Gonzalez, D. Campolo, and E. Burdet, "Differential game theory for versatile physical human-robot interaction," Nature Mach. Intell., vol. 1, pp. 36-43, Jan. 2019.

[22] M. Flad, L. Fröhlich, and S. Hohmann, "Cooperative shared control driver assistance systems based on motion primitives and differential games," IEEE Trans. Human-Mach. Syst., vol. 47, no. 5, pp. 711-722, Oct. 2017.

[23] X. Na and D. J. Cole, "Game-theoretic modeling of the steering interaction between a human driver and a vehicle collision avoidance controller," IEEE Trans. Human-Mach. Syst., vol. 45, no. 1, pp. 25-38, Feb. 2015.

[24] S. H. Tamaddoni, S. Taheri, and M. Ahmadian, "Optimal preview game theory approach to vehicle stability controller design," Veh. Syst. Dyn., vol. 49, no. 12, pp. 1967-1979, 2011.

[25] C. Dextreit and I. V. Kolmanovsky, "Game theory controller for hybrid electric vehicles," IEEE Trans. Control Syst. Technol., vol. 22, no. 2, pp. 652-663, Mar. 2014.

[26] X. Ji, K. Yang, X. Na, C. Lv, and Y. Liu, "Shared steering torque for lane change assistance: a stochastic game-theoretic approach," IEEE Trans. Ind. Electron., vol. 66, no. 4, pp. 3093-3105, 2019.

[27] R. Tian, N. Li, I. V. Kolmanovsky, Y. Yildiz, and A. R. Girard, "Gametheoretic modelling of traffic in unsignalized intersection network for autonomous vehicle control verification and validation", IEEE Trans. Intell. Transp. Syst., early access, Nov. 2020.

[28] M. Martinez-Garcia, R. S. Kalawsky, T. Gordon, T. Smith, Q. Meng, and F. Flemisch, "Communication and interaction with semiautonomous ground vehicles by force control steering," IEEE Trans. Cybern., early access, Sep. 2020.

[29] C. Li, H. Zhao, S. Zhen, and Y. H. Chen, "Control design with optimization for fuzzy steering-by-wire system based on Nash game theory," IEEE Trans. Cybern., early access, Jan. 2021.

[30] R. Li, Y. Li, S. Li, C. Zhang, E. Burdet, and B. Cheng, "Indirect shared control for cooperative driving between driver and automation in steerby-wire vehicles," IEEE Trans. Intell. Transp. Syst., early access, 2020.

[31] D. J. Cole, A. J. Pick, and A. M. C. Odhams, "Predictive and linear quadratic methods for potential application to modeling driver steering control," Veh. Syst. Dyn., vol. 44, no. 3, pp. 259-284, 2006.

[32] T. Keviczky, P. Falcone, F. Borreli, J. Asgari and D. Hrovat, "Predictive control approach to autonomous vehicle steering," in Proc. American Control Conf., Minneapolis, MN, USA, 2006.

[33] J. B. Rawlings and D. Q. Mayne, Model Predictive Control: Theory and
Design. New York: NY, USA: Nob Hill, 2009.

[34] X. Na, "Game theoretic modeling of a driver's steering interaction with active steering", Ph.D. dissertation, University of Cambridge, U.K., 2014.

[35] ISO 3888-1: Passenger cars - Test track for a sever lane-change manoeuvre - Part 1: Double lane-change, International Organization for Standardization (ISO), 1999.

[36] R. A. Auckland, W. J. Manning, O. M. J. Carsten, and A. H. Jamson, "Advanced driver assistance systems: objective and subjective performance evaluation," Veh. Syst. Dyn., vol. 46, no. 1, pp. 883-897, 2008.

[37] J. Bonnefon, A. Shariff, and I. Rahwan, "The social dilemma of autonomous vehicles," Science, vol. 352, pp. 1573-1576, Jun. 2016.

[38] L. Ljung, System Identification Theory for the User (2nd Edition). London, U.K.: Prentice-Hall, 1999.

[39] J. C. Lagarias, J. A. Reeds, M. H. Wright, and P. E. Wright. "Convergence properties of the Nelder-Mead simplex method in low dimensions," SIAM J. Optim., vol. 9, no. 1, pp. 112-147, 1998.

[40] C. MacAdam, "Application of an optimal preview control for simulation of closed-loop automobile driving," IEEE Trans. Syst., Man, Cybern., vol. SMC-11, no.6, pp.393-399, Jun. 1981.

[41] D. C. Montgomery and G. C. Runger, Applied Statistics and Probability for Engineers (7th Edition). Hoboken, NJ: U.S.A.: Wiley, 2018.

[42] J. Inga, M. Eitel, M. Flad, and S. Hohmann, "Evaluating human behavior in manual and shared control via inverse optimization," in proc. IEEE Int. Conf. Syst., Man, and Cybern., Bari, Italy, pp. 2699-2704, 2018.

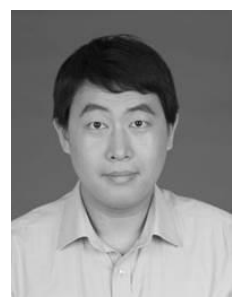

Xiaoxiang Na received the B.Sc. and M.Sc. degrees in automotive engineering from the College of Automotive Engineering, Jilin University, China, in 2007 and 2009, respectively. He received the Ph.D. degree in drivervehicle dynamics from the Department of Engineering, University of Cambridge (CUED), U.K. in 2014, where he is currently a Senior Research Associate.

His main research interests include driver-vehicle dynamics, in-service monitoring of heavy goods vehicle operations and assessment of vehicle energy performance.

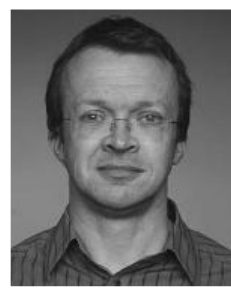

David J. Cole received the B.A. degree in engineering and the Ph.D. degree in vehicle dynamics from the Department of Engineering, University of Cambridge (CUED), U.K. in 1985 and 1990, respectively.

From 1990 to 1996, he undertook his postdoctoral research in heavy vehicle dynamics at CUED, where he is currently a University Professor. From 1996 to 2000, he was a Lecturer with the University of Nottingham, U.K. In 2000, he returned to CUED to take up his present position. His main research interests include driver-vehicle dynamics. $\mathrm{He}$ is a Chartered Engineer and a Fellow of the Institution of Mechanical Engineers. 